\title{
Artelogie
}

Recherche sur les arts, le patrimoine et la littérature de l'Amérique latine

1 | 2011

Brésil, questions sur le modernisme

\section{Le désir d'Amérique : De la nostalgie occidentale à l'affirmation du métissage américain : trois artistes d'Amérique latine en France}

\section{Christine Frérot}

\section{(2) OpenEdition}

Édition électronique

URL : https://journals.openedition.org/artelogie/8751

DOI : 10.4000/artelogie.8751

ISSN : 2115-6395

Éditeur

Association ESCAL

Référence électronique

Christine Frérot, « Le désir d'Amérique : De la nostalgie occidentale à l'affirmation du métissage américain : trois artistes d'Amérique latine en France », Artelogie [En ligne], 1 | 2011, mis en ligne le 01 mars 2011, consulté le 07 janvier 2022. URL : http://journals.openedition.org/artelogie/8751 ; DOI https://doi.org/10.4000/artelogie.8751

Ce document a été généré automatiquement le 7 janvier 2022.

Association ESCAL 


\title{
Le désir d'Amérique : De la nostalgie occidentale à l'affirmation du métissage américain : trois artistes d'Amérique latine en France
}

\author{
Christine Frérot
}

«Aucun continent au monde n'a provoqué, sur des millions de lèvres et avec autant d'insistance, la naissance du mot « Paradis ». Il n'a jamais été prononcé à l'endroit de l'Afrique quand elle fut découverte, ni de l'Asie, cette terre morcelée en îlots et en archipels. Aucun continent n'a suscité une littérature aussi vaste et riche : romans, poèmes, drames, tragédies, comédies, récits de voyages, chroniques, carnets de bord, rapports de soldats et d'officiers, journaux, lettres etc... Toute une littérature va dire que l'Amérique est un paradis. (...) C'est cette notion de paradis qui

pèsera d'un poids décisif sur notre sensibilité européenne et qui créera ce lien si particulier que nous entretenons depuis le 12 octobre 1492, avec

l'Amérique ".

Berger Yves, Propos recueillis par Gérard de

Cortanze, Magazine Littéraire $\mathrm{n}^{\circ} 296$ «1492,

l'invention d'une culture », Paris, Février 1992.

1 Depuis le XVIème siècle, historiens et écrivains n'ont eu de cesse de vouloir expliquer la découverte de l'Amérique et de débattre de la question de son attribution à Colomb. Leurs hypothèses et leurs arguments sur les causes et les raisons du voyage du Génois, autant liés aux mythes (Jardin des Hespérides, Atlantide ou Eldorado) qu'à la religion (l'inspiration de Dieu et l'expansion du christianisme), au commerce (chercher une 
autre route vers l'Asie) ou à l'histoire (l'homme est le seul responsable de son projet scientifique), sont aussi traversés par des spéculations de tout ordre qui influeront sur le contenu des représentations iconographiques.

Les idées avancées dans leurs grands récits par Oviedo, Sahagún, Bartolomé de las Casas ou plus près de nous Alexander Von Humboldt -pour ne citer que ceux-là-, ont jalonné les siècles et imprégné les consciences, ont tissé une mémoire collective où s'enracine l'idée de l'Autre - du différent- dont va hériter l'Occident dès la Renaissance. A partir d'une brillante exégèse des textes de ces auteurs ${ }^{1}$, l'historien mexicain Edmundo O'Gorman avance le concept d'une Amérique inventée au lieu d'une Amérique découverte ${ }^{2}$, idée qui sous-tend une autre conception du monde. Jusqu'en 1492, la vision chrétienne imposait sa trilogie exclusive et unique : l'Europe, l'Asie et l'Afrique. Avec la « découverte » d'une « quatrième partie » (l'Amérique) -différente mais proche de l'Europe et que la tradition scientifique et religieuse n'avait pas prévu-, se fait jour l'idée d'une autre Europe, celle de un «nuevo mundo » qui deviendra alors synonyme d'Amérique. Mais la perte du « Paradis terrestre » chrétien subsistera à travers le temps dans l'inconscient individuel et collectif, nourrissant de "désir d'Amérique " l'iconographie allégorique et utopique des artistes européens; de leur côté, avec d'autres aspirations et d'autres servitudes, les artistes de l'Amérique latine voudront construire une identité américaine. Avant et après la Conquête, l'Amérique latine n'a cessé de susciter et de cristalliser en Europe des passions autant que des peurs, créant des images irrationnelles et des mythes autour d'un continent à la fois mystérieux, fascinant et effrayant ${ }^{3}$. Les attentes où se conjuguent recherche d'aventure, envies de nature vierge et besoin d'infini vont entrainer la formation des stéréotypes qui vont alimenter la création artistique et nourrir les mémoires vives, contribuant ainsi à la construction d'un regard, changeant et néanmoins immobile, sur l'Amérique. Soif d'étrangeté ou aveuglement mimétique, le regard porté sur les artistes d'Amérique latine se dessine et varie plutôt selon le contenu que la forme des œuvres, mais aussi en fonction des idées dominantes d'une époque. Quant aux préoccupations existentielles des artistes latino-américains, elles conjuguent identité et esthétique et sont imprégnées d'une vision personnelle ou collective de leur continent qui a aussi à voir avec un autre " désir d'Amérique ». Observer l'œuvre des artistes latino-américains en France à partir de ce dernier postulat, c'est se placer d'un côté du point de vue des conquistadores - ce sera le cas pour Pedro Figari - et de l'autre, de celui des artistes, les conquistados - ce sera le cas pour Tarsila do Amaral et Wifredo Lam. Dans cette observation complexe de la nature d'une Amérique désirée, confluent et se croisent des considérations, des références, des analogies ou des extrapolations vis à vis de «l'ailleurs $»^{4}$, de « l'exotisme $»^{5}$, des «territoires absents $»^{6}$ et de « l'entre-deux $»^{7}$. C'est en 1907 qu'Henri Rousseau dit Le Douanier (1844 - 1910), peintre français autodidacte, a peint son tableau emblématique "La charmeuse de serpents $»^{8}$, scène mystérieuse dont l'énigme des origines laisse parfois planer le doute d'une vision inspirée de la réalité ou de celle d'un monde imaginaire. Quel qu'en soit le modèle, réel ou rêvé, c'est l'atmosphère primitive et exotique du tableau qui fascine alors autant Picasso que Gauguin et les conforte dans leur approche esthétique des cultures dites primitives. Cet hommage onirique et serein au Paradis perdu ou à l'Eden n'est en fait rien d'autre qu'une réponse utopique bien que flamboyante de ces désirs d'ailleurs toujours renouvelés dont l'Amérique sera, après l'Egypte et le Japon, la terre de prédilection même si, à ce moment-là, les cultures amérindiennes sont à peine connues ou carrément ignorées face à la découverte de l'art africain. Mais comme l'écrit Gilles 
Manceron dans sa préface à 1 '" Essai sur l'exotisme » de Victor Segalen", « l'exotisme était à la mode dans les deux premières décennies du XXème siècle, qui, plutôt que l'ouverture d'un siècle nouveau comme les millésimes porteraient à la croire, étaient au contraire l'apogée et les derniers spasmes d'une ère qui s'achevait, celle en particulier de l'épopée coloniale et de la toute-puissance européenne ». Les écrivains dans leurs romans, les peintres avec leurs toiles et les commentateurs avec leurs critiques, cultivent le thème de l'exotisme qu'ils associent au dépaysement géographique et aux impressions du voyage touristique dans un attrait superficiel et folklorique des cultures autres. A propos des artistes d'Amérique latine, le vocabulaire des textes critiques relevés dans la presse française des années 20 et 30 est révélateur de cette soif de nouveautés lointaines, avec cet amalgame réducteur qui se fait entre le primitif, le local, l'origine, la terre, le naturel..., et l'exotique. Pourtant, au même moment, un climat nationaliste identitaire entretient une xénophobie qui s'exprime ouvertement face à la présence croissante des artistes étrangers. Ces derniers sont qualifiés de métèques qu'on accuse de pulluler dans les rues et les Salons ${ }^{10}$; ils sont accusés de faire subir au milieu de l'art français «la pression d'une invasion étrangère sans passé artistique, sans esthétique, et, à vrai dire, purement barbare $»^{11}$ dont les œuvres menacent l'art français et risquent de le dénaturer. Cette différence culturelle qui est abhorrée et perçue comme une menace est reçue par d'autres comme un apport novateur qui doit régénérer un art trop intellectualisé (aux yeux de ses détracteurs, le cubisme) et s'imposer face à une internationalisation à laquelle devraient -selon euxrenoncer les artistes d'Amérique latine. Mais qu'attend-t-on réellement des artistes latino-américains? Dans un texte de 1947, le critique Georges Pillement, grand admirateur de Figari, explique que ces derniers doivent savoir évoquer « l'atmosphère de leur pays » et "la nature américaine $»^{12}$; d'autres ${ }^{13}$, avec un ton condescendant, paternaliste et néo-colonial, s'enthousiasment pour les «traditions", le «souci décoratif proprement aztèque ", la " géométrie fleurie » de certains qu'ils qualifient de « véritables primitifs » dont, cette fois, « l'invasion est pacifique ». L'Uruguayen Pedro Figari va constituer lors de son séjour parisien le paradigme de ce regard exotique qui se confond avec le désir d'Amérique.

\section{Le paradigme américain de l'exotisme : Pedro Figari}

3 Avocat et homme politique, défenseur des droits de l'homme, Figari (Montevideo 1861 1938) peint d'abord pendant ses loisirs, puis professionnellement à partir de 1921 : il a alors 60 ans. C'est à Paris entre 1925 et 1934 qu'il va réaliser l'essentiel de son œuvre (plus de 2000 toiles) en partant de sa théorie du « régionalisme artistique », élaborée dès 1912, et qui revendique un objectif et un destin à sa peinture : élever le niveau de la culture dans son pays. Figari peint essentiellement des patios coloniaux, des fêtes où sont présents les Noirs, des danses et des chants populaires, toute l'atmosphère de la Pampa de la fin du 19ème siècle. (El patio, huile sur carton, 67,5 x 98 ; La novia, ; huile sur carton, 49 x 79) ; Candombe, huile sur carton, 32,5 x 39,5). Alors que les artistes étrangers qui choisissent la France sont en quête de nouveauté et de modernité, Figari décline en images sa mémoire géographique et culturelle, celle qui le relie à l'enfance, avec des accents post-impressionnistes à la Vuillard et à la Bonnard. En décalage avec une époque où se côtoient, en s'opposant, Surréalistes et défenseurs de l'Abstraction et du post-cubisme, il va susciter -hors de son pays ${ }^{14}$ - un engouement sans limites qui en fera le premier artiste d'Amérique latine à jouir d'une monographie en France ${ }^{15}$ et celui 
sur lequel on trouve le plus de textes critiques (la majorité d'entre eux élogieux) au cours des années 20. Situation paradoxale où un peintre plutôt moyen vient cristalliser cette envie d'Amérique en proposant des œuvres qui représentent un "autre ", un " ailleurs » désirables parce que l'artiste est lui-même respectable de part ses origines et que son art, qui ne remet ni la modernité ni la tradition picturale en question, est acceptable et recevable par tous. Si d'autres peintres d'Amérique latine ont ouvert des voies singulières et trouvé leur place à Paris comme le mexicain Diego Rivera ou le Brésilien Vicente Do Rêgo Monteiro alors qu'un autre uruguayen, Joaquin Torres Garcia, tentait d'imposer, sans succès, sa conception américaine d'un art construit, c'est à propos de Figari que l'on écrit en $1923^{16}$ " qu'il est le seul peintre américain qui peigne en Américain ». Assertion reprise soixante-dix ans plus tard dans un texte écrit à propos de la dernière exposition Figari à Paris en 1992 où l'un des auteurs du catalogue, Nelson Di Maggio ${ }^{17}$, insiste à nouveau sur la " foi américaniste » de Figari, à une époque où il «fallait faire renaître le sentiment américain naturel». Dans un climat de déshumanisation de l'art, l'art de Figari, écrit Jean Cassou $^{18}$, est " pureté de l'émotion ", « don des Dieux, « merveilleuse innocence », créé par un « homme sensible et naturel». Raymond Cogniat ${ }^{19}$ surenchérit dans ce registre idéalisé en parlant de l'art de Figari comme d'une "fantaisie charmante», une peinture de "l'anecdote» pleine de « conviction » et de « foi ». Le travail de mémoire du peintre est salué avec nostalgie : «Figari est en train d'écrire le poème de la race (...), le poème de tant de choses qui ont été et qui ne seront plus $»^{20}$. Il devient le chantre de la "recherche du temps perdu $»^{21}$ et le texte de Georges Pillement de 1930 cherche à en faire le porte-parole de l'affirmation de l'identité. Ce dernier parle de "réveil de l'Amérique latine " en mentionnant les artistes mexicains et ceux du Rio de la Plata dont surtout Figari. L'Uruguayen semble donc être celui qui a réussi à évoquer sans revendication révolutionnaire et sans violence iconographique sa terre et ses coutumes, à représenter les Noirs et leurs moeurs locales, à faire rêver, -il déclare lui-même que c'est le rêve qui préside à son acte de création -, sur un passé révolu mais implicitement regretté où règnent plaisir, distraction et nature toute-puissante; Amérique d'hier recréée aujourd'hui dont l'évocation a de quoi alimenter les désirs de dépaysement avec ses images séduisantes amalgamant le « national » et « l'exotique » dans un climat parisien où s'exacerbent les antagonismes non seulement esthétiques mais idéologiques. "Emotion", "gaucherie ", "répétition", réduction à une vision de l'Amérique illustrative et fantaisiste, sont les reproches ${ }^{22}$ faits à Figari, qui comme on a pu le voir plus haut sont, pour d'autres, des qualités. Enfin, c'est sur la peinture de Figari que se portent tous les espoirs, c'est en elle que se cristallisent et se fixent tous les désirs d'une société proche de la nature, respectueuse et soucieuse de son histoire et de son passé, la seule qui puisse générer un progrès. Pour Jean $\mathrm{Cassou}^{23}$ « il est le meilleur, en tout cas le plus apte à soutenir dans son entreprise une race jeune, neuve, soucieuse de raison et de liberté, naturellement démocratique, véritablement initiatrice, fondatrice (...) Cette race, répétons-le, se souvient de ses origines, elle porte en elle l'énergie de la nature primitive. (...) Le primitif est pour lui (Figari) la condition même du progressif ». Pour Jules Supervielle, la peinture de Figari exalte « les souvenirs de l'enfance d'un poète qui avait su donner au pittoresque sa raison d'être et sa profondeur ». 


\section{Tarsila do Amaral et la gestation parisienne d'une identité brésilienne}

4 Comme pour les étudiants en art et les peintres de sa génération, le voyage à Paris facilité par son appartenance à l'oligarchie terrienne brésilienne-, est pour Tarsila do Amaral (1890 - 1973) l'indispensable passage pour aborder les nouveaux courants qui fondent l'art moderne. Les motivations de son départ sont différentes de celles de Figari : Tarsila veut se former et adhérer à la modernité européenne, y être reconnue et conquérir une place enviable sur la scène parisienne ; ensuite, forte de cette expérience européenne, retourner au Brésil afin de s'imposer dans le monde de l'art de son pays. Elle n'est alors pas encore préoccupée par l'existence et la formulation d'une possible «brésilianité » artistique, à l'opposé d'un Figari profondément animé du désir de conserver et de faire revivre, avec son art, les traditions de son pays.

5 Mais plusieurs évènements vont modifier le contenu des projets de Tarsila dont Paris sera le déclencheur. Entre 1920 et 1923, elle peint des nus, plutôt traditionnels, à l'Académie Julian. Consciente que son apprentissage de la modernité est lié au cubisme, elle entre dans l'atelier d'André Lhote puis surtout dans celui de Fernand Léger. Elle va alors s'ouvrir à des formes nouvelles dont l'influence sera rapidement perceptible dan son œuvre. Au même moment le Brésil joue une carte majeure pour l'avenir de l'art non seulement brésilien mais latino-américain. La "»Semana de arte moderno " à Sao Paulo en Février 1922 est un évènement capital, circonscrit aux élites intellectuelles et à l'avant-garde artistique, où seront regroupés littérature, théâtre, peinture, sculpture et architecture, pour une rupture avec l'académisme et la tradition. Un de ses animateurs, le poète Oswald de Andrade, publie en 1924 le « Manifeste de la Poésie Pau Brésil » et en 1928 le «Manifeste Anthropophage». Ces deux textes fondent l'esprit anthropophage dans lequel va dorénavant s'inscrire la création brésilienne : exalter un Brésil riche de ses traditions sans négliger l'apport de la modernité occidentale; cristalliser le désir d'une identité brésilienne articulée sur l'assimilation de deux expériences, de deux savoirs. Inventer un art national et ne plus copier l'étranger. Tarsila accompagne directement ce mouvement avec sa peinture en comprenant que l'attrait que les Européens ont pour le primitif peut justifier la découverte de sa propre culture. Tarsila, qui est devenue la compagne d'O. de Andrade, a découvert la culture populaire de son pays lors d'un voyage avec le poète Blaise Cendrars.

6 L'œuvre audacieuse et emblématique du changement qui s'opère en elle est incontestablement A negra, huile sur toile, 100 x 81,3, peinte à Paris en 1923, où sont exaltées (et exagérées dans la forme) les origines raciales, plutôt africaines, du Brésil ; œuvre mythique, figure hybride qui montre sa recherche de "brésilianité ». Au cours des années de présence à Paris, le travail de Tarsila do Amaral est à la pointe des idées du manifeste anthropophage car elle est l'artiste la plus liée au groupe moderniste. Tous les enseignements dont témoignent sa peinture, du constructivisme au surréalisme en passant par Léger et le Douanier Rousseau, concourent à l'exaltation d'un Brésil simultanément traditionnel et contemporain illustré par des peintures aux thèmes brésiliens réalisées à Paris : Estaciao central de ferrocarril do Brasil, huile sur toile, 1924, 142 x 126cm et Morro da favela, huile sur toile, 64 x 76, 1924, qui avec A negra, font partie des œuvres présentées à sa première exposition personnelle Galerie Percier, en 1926.24. Si la volonté d'inscription de Tarsila et Oswald de Andrade dans le milieu artistique français s'explique par une recherche de légitimation de leur « découverte » 
du Brésil noir et indien -le recours au primitivisme en art étant fort valorisé par les Cubistes et les Surréalistes- il n'en reste pas moins qu'ils sont les aspects d'une stratégie de conquête que Paulo Herkenhoff ${ }^{25}$ qualifie «d'anthropophagie de l'élite ». En effet, dans les œuvres postérieures brésiliennes de Tarsila, dont la dimension primitive est plus proche d'une vision naïve et édulcorée de la réalité que d'un regard critique distancié, n'apparaît aucune dimension sociale. Comme l'explique Elsa Ajzenberg ${ }^{26}$ «l'accueil que la France réservait aux cultures « exotiques » comme celles d'Afrique et de Polynésie, fut un acteur revivifiant pour son art. Il entraîna une valorisation internationale des forces indigènes contribuant à induire divers artistes brésiliens à rechercher l'expression du «national» dans lequel l'exotique était un facteur naturel. Ce contexte constituera l'axe moteur de la démarche créatrice de Tarsila ».

\section{L'œuvre de Lam propose l'Amérique latine du métissage}

7 Plusieurs artistes d'Amérique latine nous ont donné une leçon de métissage des formes et des images et ont été les modèles de l'ouverture à la culture de l'autre et à son assimilation. Certains ont été admirés et compris par les poètes qui ont abondamment écrit sur eux, d'autres un peu méprisés par les tenants d'une conception de l'art, moins humaniste et plus rationnelle, mettant surtout en avant des valeurs conceptuelles. Des maitres comme le Cubain Wifredo Lam, ou plus récemment le Mexicain Francisco Toledo, qui ont élaboré des langages mixtes intégrant simultanément leurs racines culturelles et plusieurs courants de l'art moderne, ont contribué à ouvrir un dialogue entre Amérique et Europe occidentale, faisant accepter leurs brassages comme modernes et relativisant par là-même le schéma bi-polaire entre centre et périphérie.

8 L'oeuvre de Lam poursuit ce besoin occidental d'Amérique, mais son art, sans nostalgie ni anecdote (Figari), sans stratégie de conquête (Tarsila), s'inscrit dans une esthétique identitaire marquée par l'influence de Picasso, inspirée par l'Afrique et traversée intimement par l'expérience culturelle de la conscience de soi. En inventant un monde que l'on dira magique, dans lequel les poètes découvriront des échos de création, l'artiste impose son propre regard sur son continent métissé et fait reculer le regard exotique extérieur. Wifredo Lam (1902 - 1982) est né à Cuba d'un père chinois et d'une mère mulâtre. A Paris où il arrive d'Espagne en 1938, côtoyant Picasso autant que les Surréalistes, il découvre l'art de l'Afrique, appelé « art nègre ». Mais ce n'est qu'en 1941, lorsqu'il rentre aux Antilles (Haïti et Cuba) que va se produire le choc qui va ébranler définitivement son être intime autant que sa personnalité artistique. L'influence prégnante de Picasso est balayée, il ne reste dans ses nouvelles images qu'une mémoire schématisée de la désarticulation de la figure qu'il va mettre au service de la résurgence de ses racines africaines pour exprimer mythes et rituels, violence et douceur, nuit et lumière. "Le sorcier de l'Océan », huile sur toile, 107 x 85, 1947 ; «Le Tiers-Monde », huile sur toile, 251 x 300, 1966.Dans son œuvre « La Jungle », gouache sur papier marouflé sur toile, $239,4 \times 229,9,1943^{27}$, où l'Afrique est convoquée dans sa dimension politique, où les images archétypales du passé sont aussi des métaphores du présent, où l'imaginaire et la réalité régionales se confondent, Lam s'impose car il exprime, dans ce nouveau langage, le désir d'un monde meilleur où s'entrechoquent et s'entrelacent les différences. Si d'un côté on lui reproche d'exalter le métissage, de l'autre, pour cette même raison, il est considéré comme le sauveur de notre humanité 
en perdition. "Wifredo Lam résout peut-être le grand vœu de notre vingtième siècle qui se meurt de ne pouvoir fondre dans un alliage idéal la connaissance transcendante du primitif et la science positive du civilisé $»^{28}$ écrit-on en 1946 à l'occasion de son exposition. Lam se sait profondément investi de ce nouveau pouvoir d'invoquer et d'associer l'ancestral et l'actuel, le primitif et le moderne, le rêve et le réel dans sa conception et sa vision de l'Amérique; il est relayé dans cette quête par les poètes, notamment Aimé Césaire, pour qui il devient une sorte de magicien qui «célèbre la transformation du monde en mythe et en connivence " ${ }^{29}$.Par "son instinct natal ", enrichi du « don de l'intuition primitive ${ }^{30}$, Lam est un « restaurateur de rites », et son œuvre lutte contre la raison, l'égoïsme, l'angoisse car elle est universelle ${ }^{31}$. Lam est non seulement Américain et Européen, mais il est au-delà de toutes frontières. «Et c'est précisément cet exceptionnel mélange de réalités lointaines, ce jeu d'antagonismes perpétuel qu'assument son esprit et son corps, qui lui permet de trouver, dans son œuvre, des termes généraux, qui peuvent avoir un sens à la fois pour un Européen, un Américain, pour un Extrême-Oriental ou pour un Africain " ${ }^{32}$. Les "visions magiques ${ }^{33}$, la dimension cérémonielle, mélodique de son oeuvre, le rythme et la liberté ${ }^{34}$ qui l'imprègnent la rendent proprement américaine. C'est ce que souligne dès $1948^{35}$, un autre américain nourri de culture française, l'écrivain cubain Alejo Carpentier. Pour lui, Lam est l'artiste du « réel-merveilleux américain » qui célèbre ce qui en lui est proprement américain et que ne peuvent atteindre les Surréalistes, à ses yeux artificiellement englués dans la sophistication et l'intellectualité qui paralyse leurs velléités d'imaginaire. Lam, un peintre latino-américain qui propose enfin la première vision non exotique de l'Amérique et fait exister culturellement un continent dans son hybridité. Comme le souligne Aimé Césaire ${ }^{36}$ «il fallait rompre avec les puissants amateurs de cartes postales (...). La peinture de Lam arrête la geste du conquistador ; elle signifie son échec à l'épopée sanglante de l'abâtardissement par son affirmation insolente qu'il se passe désormais quelque chose aux Antilles ».

\section{Conclusion}

9 Le regard se normalise internationalement tout en maintenant une relative polémique entre local et global. Comme l'écrit Stuart Hall ${ }^{37}$ « la globalisation ne doit jamais être lue comme un simple processus d'homogénéisation culturelle: c'est toujours une articulation du local, du spécifique et du global». L'ébranlement du regard eurocentriste sur l'art de l'Amérique latine se produit avec l'avènement de l'art cinétique à Paris dans les années 60 conduit par ses leaders latino-américains. Cette génération qui va créer la distance avec un continent circonscrit dans le regard européen à une figuration souvent "folklorique ", inaugure une nouvelle esthétique, utilise de nouveaux matériaux et des techniques souvent plus en phase avec la technologie et la réception mondialisée qu'avec un quelconque objectif latinoaméricaniste. La rupture se produit dans les deux sens puisque s'est estompée en France cette fascination pour une Amérique rêvée et inventée qui sera bientôt remplacée par la terrible réalité des dictatures (années 70). Plus tard, avec ses artistes exilés - surtout Chiliens, Argentins et Brésiliens -, l'art de l'Amérique latine rejoint celui des artistes post-soixante-huitards dans le sillage d'une figuration narrative française, réaliste et politique (Les Malassis). La réception ira de soi à ce moment-là. Les exilés seront accueillis, reconnus et invités à exposer. 
10 Mais le vrai changement dans notre vision de l'art de l'Amérique se produit dans les années 80 avec l'internationalisation de l'art ou plutôt le déplacement de ce que l'on appelait jusqu'alors les centres et les périphéries. De nouvelles métropoles surgissent où l'art de l'Amérique latine se montre, circule, s'impose; de nouvelles instances sont crées dans les pays dits du Sud: les Biennales de la Havane, Johannesburg, Kwanju, Istamboul....) où l'art de l'Amérique latine devient de "l'art international ", où les artistes comme les autres acteurs du monde de l'art se globalisent. Quel est le regard porté à ce moment-là sur l'art de l'Amérique latine ? Quelles attentes induit-il, quelles idées s'en fait-t-on? Les grandes manifestations comme celle de 1992 témoignent encore de l'enracinement de ce concept de différence, non pas dans sa valeur ontologique, mais plutôt en tant que différent par rapport à l'art dit occidental. Donc une différence relative et non absolue. L'article de Philippe Dagen paru dans le journal Le Monde à l'occasion de l'exposition au Centre Georges Pompidou en 1992 montre que les clichés sont bien enracinés vis à vis de l'art de l'Amérique latine, continent dont l'art se définit à ses yeux par la lenteur et le mimétisme, l'auteur utilisant la métaphore de la tortue et du caméléon. Les verrous sauteront avec la multiplication des expositions individuelles en Europe - notamment avec celles du Mexicain Gabriel Orozco, du Brésilien Ernesto Neto, du cubain Kcho ou de l'Argentin Guillermo Kuitcaqui modifieront la réception qualitative de l'art de l'Amérique latine et les visées nationalistes de son exportation. Avec la globalisation, l'exacerbation des questionnements sur la nationalité et l'hybridité se fait jour. On peut alors se poser, comme l'a fait Serge Gruzinski ${ }^{38}$ dans "la Pensée métisse ", la question de savoir si l'hybride est finalement en train de détrôner l'exotique.

\section{BIBLIOGRAPHIE}

\section{Ouvrages cités}

CARPENTIER, Alejo. Chroniques, Paris : Gallimard, 1983.

CURZI, Lucien. Lam, Bologne (Italie) : Bora, 1978.

GRUZINSKI, Serge. La Pensée métisse, Paris : Fayard, 1999.

LE CLEZIO, Jean-Marie. Ailleurs, Paris : Arléa, 1995.

O'GORMAN, Edmundo. La invención de América, Mexico: Lecturas Mexicanas 63 Fondo de Cultura Económica/Sep, 1958.- PILLEMENT Georges. Pedro Figari, Paris : G. Crès et Cie, 1930.

ROJAS MIX, Miguel. America imaginaria, Barcelona: Lumen, 1992.

SEGALEN, Victor. Une esthétique du divers, Paris : Fata Morgana, 1978.

SIBONY, Daniel. Entre-deux, l'origine en partage, Paris : Seuil, 1991. 


\section{NOTES}

1. La invencion de América, Lecturas mexicanas 63, Mexico, 1958, 193p.

2. op. cit. p. 54.

3. Comme l'a décrit Miguel Rojas Mix dans America Imaginaria , Lumen éd., Barcelona 1992, 251 p.

4. Celui qu'évoque Jean-Marie Le Clézio dans Ailleurs, entretiens avec Jean-Louis Ezine, Arléa éd., Paris, 1995, 125p.

5. Victor Segalen, Une esthétique du divers, Victor Segalen (oeuvre posthume), Fata Morgana éd., 184p. lère édit. en 1955.

6. Gerardo Mosquera, texte du catalogue de l'exposition Territorios ausentes, Casa de America, Madrid, 27/1 - 26/3/2000.

7. Daniel Sibony, Entre-deux, l'origine en partage, Seuil édit., Paris 1991, 399 pages.

8. Paris, Musée d'Orsay.

9. Victor Segalen, op. cit., p. 9.

10. Camille Mauclair, «Une campagne picturale ", La Farce de l'art vivant I, éditions de la Nouvelle revue critique, Paris 1929, 215p. et «Les métèques contre l'art français », La Farce de l'art vivant II, éditions de la Nouvelle revue critique, Paris 1930, 214p.

11. Georges Rivière, "Avons-nous encore un art français?", L'Art Vivant, Tribune libre du ler septembre 1930, p. 721-722.

12. Antonio Terry, Revue de l'Amérique latine, Paris, Juillet 1929 (article sur Figari), p. 81.

13. F.M., "On demande de la peinture de sauvages », Bulletin de la vie artistique, ler Août 1926, $\mathrm{n}^{\circ} 15$

14. En Uruguay où son travail suscite d'abord un rejet, car on considère sa quête des traditions comme une attitude passéiste.

15. Georges Pillement, Pedro Figari, coll. «Les artistes nouveaux », éditions G. Crès et Cie, sous la direction de Georges Besson, 32 reproductions, Paris, 1930.

16. Charles Lesca, « Pedro Figari, peintre uruguayen », Revue de l'Amérique latine, Paris, avril 1923, p. 166-167.

17. Pavillon des Arts 5 mars -2 mai 1992, catalogue p. 42.

18. Revue de l'Amérique latine, «L'art de Figari », Paris, mars 1926, p. 259 -261.

19. Revue de l'Amérique latine, Paris, décembre 1923, p. 357-358.

20. Idem op. cit.

21. G. Guillot-Munoz, «La peinture de Pedro Figari », Revue de l'Amérique latine, Paris novembre 1925, p. 403 à 406.

22. Waldemar Georges, «A propos de l'exposition Galerie Druet et François Fosca, " L'Amour de l'art, Paris 1925. «A propos de l'exposition Galerie Druet », L'Amour de l'art, Paris 1927.

23. Exposition Pedro Figari, Musée National d'Art moderne, catalogue avec textes de Jules Supervielle et Jean Cassou (sans pagination), Paris 1960.

24. Texte dans le catalogue de Blaise Cendrars intitulé "Sao Paulo ».

25. Catalogue de l'exposition "Tarsila do Amaral, Paris 1923 - 1929 », Maison de l'Amérique latine, Paris 15 décembre 2005 - 20 février 2006, p. 51.

26. Idem op. cit. p. 68

27. Le tableau se trouve aujourd'hui au Museum of Modern Art de New-York.

28. Revue Arts, ler mars 1946, non signé.

29. Aimé Césaire, Cahiers d'Art, 1945-1946, p. 357.

30. Lucien Curzi, Lam, éditions Bora, Bologne, Italie, 1978, p. 28.

31. Alain Jouffroy, « Cuba », L’ARC n 23, automne 1963, p. 79.

32. Idem Alain Jouffroy, op. cit.

33. Nicolas Calas, « extrait de « Magic Icons », Horizont, Londres vol. XIV, 1946.

34. Pierre Mabille, Cuadernos Americanos, Mexico 1944. 
35. Le réel-merveilleux en Amérique, Chroniques, Gallimard, coll. Idées, Paris 1983, p. 342-349.

36. Op. cit . p. 357.

37. «Representation, cultural representation and signyfing practices" (1997) cité dans Expériences du divers, p. 12, collection "métiers de l'exposition », éditions Presses Universitaires de Rennes, 2000, 151 pages.

38. Paris, éditions Fayard, 1999, p. 48.

\section{RÉSUMÉS}

D'un indéniable désir d'Amérique (le paradis perdu, la nature vierge, l'exotisme...) à l'exigence d'un art engagé nourrie par les exilés des dictatures, le regard porté sur l'art de l'Amérique latine en France a subi plusieurs transformations. Un Uruguayen, Joaquín Torres García, propose au début des années 30 une alternative à l'avant-garde occidentale, dans la version à la fois américaine et universelle d'une géométrie influencée par Mondrian, alors que règne à Paris un climat hostile envers ceux que l'on considère comme des "métèques ». En dehors des poètes surtout ceux liés au surréalisme - et certains critiques qui reconnaissent à partir des années 40 la valeur d'altérité de plusieurs artistes (dont Matta, Tamayo et Lam), il faudra attendre les années 60 pour que s'ébranle le schéma bi-polaire (narration et engagement) d'un regard essentiellement eurocentriste et que se modifie lentement de la réception de l'art latinoaméricain en France. Plusieurs artistes installés à Paris (comme Julio Le Parc, Jésus Soto et Carlos Cruz-Diez) tiennent une place décisive dans ce nouveau mouvement qu'est l'art cinétique. C'est seulement au cours des années 90 que l'on observe un vrai changement lorsque l'art latinoaméricain s'impose internationalement dans l'expression et la revendication de ses particularités. C'est dans une modernité paradoxale et assumée, entre (village) local et (village) global, qu'il s'installe avec une grande liberté dans le passé et le présent, entre identité traditionnelle et altérité moderne. Aujourd'hui, la mondialisation engendre une uniformisation de la production artistique. Paris n'y échappe pas et depuis une dizaine d'années, l'art latinoaméricain y est reçu dans les galeries ou dans des expositions collectives selon une perspective critique normative qui répond aux exigences du marché international. Pourtant, c'est bien sa diversité d'origines et de cultures qui lui a permis de s'imposer et de transcender les questions de territoire et de lieu pour dépasser la simplification d'un débat Nord-Sud, en créant un espace artistique sui-generis, celui de « l'entre-deux », en rejetant la simplification des oppositions et en écartant l'idée de deux mondes qui s'affrontent.

\section{INDEX}

Mots-clés : Désir, art latino-américain, reception, Paris 


\section{AUTEUR}

\section{CHRISTINE FRÉROT}

Christine Frérot est docteur en sociologie et sémiologie des arts et des littératures de l'Ecole des Hautes Etudes en Sciences sociales, Paris (1980). Spécialiste de l'histoire de l'art latino-américain moderne et contemporain, elle a publié deux livres sur l'art mexicain : « El mercado del arte en México 1950-1976 « éditions INBA, 227 pages, Mexico 1990 et « Echanges artistiques contemporains, la France et le Mexique », éditions L'Harmattan, 159 pages, Paris 1996. Après son ouvrage « Mexico Mosaïque, Portraits d'objets avec ville », éditions Autrement, 165 pages, Paris 2000, elle a publié : « Art contemporain d'Amérique latine, Chroniques françaises 1990 - 2005 », éd. l'Harmattan, 356 pages, Paris 2005. "Resistencia visual, Oaxaca 2006", éditions Talmart, 91 pages. ill. Paris 2009. Critique d'art, membre de l'AICA, elle collabore à la revue internationale Art Nexus (Bogota-Miami) et rédige régulièrement articles et préfaces de catalogues. En tant que commissaire, elle a organisé près de cinquante expositions. Elle effectue actuellement des recherches sur l'art latino-américain en France au XXème siècle (groupe Efisal/Cral à l'EHESS, Ecole des Hautes Etudes en Sciences Sociales). Elle est également chargée de cours en histoire de l'art latino-américain à l'Université de Paris III (Censier et IHEAL, Institut des Hautes Etudes de l’Amérique latine) 